\title{
Morphological Analysis of the Left Ventricular Endocardial Surface and Its Clinical Implications
}

\author{
Anirban Mukhopadhyay ${ }^{1}$, Zhen Qian ${ }^{2}$, Suchendra M. Bhandarkar ${ }^{1}$, Tianming Liu ${ }^{1}$, \\ Sarah Rinehart ${ }^{2}$, and Szilard Voros ${ }^{3}$ \\ ${ }^{1}$ Department of Computer Science, The University of Georgia, Athens, GA, USA \\ ${ }^{2}$ Piedmont Heart Institute, Atlanta, GA, USA \\ ${ }^{3}$ Stony Brook University Medical Center, Stony Brook, NY, USA
}

\begin{abstract}
The complex morphological structure of the left ventricular endocardial surface and its relation to the severity of arterial stenosis has not yet been thoroughly investigated due to the limitations of conventional imaging techniques. By exploiting the recent developments in Multirow-Detector Computed Tomography (MDCT) scanner technology, the complex endocardial surface morphology of the left ventricle is studied and the cardiac segments affected by coronary arterial stenosis localized via analysis of Computed Tomography (CT) image data obtained from a 320-MDCT scanner. The nonrigid endocardial surface data is analyzed using an isometry-invariant Bag-ofWords (BOW) feature-based approach. The clinical significance of the analysis in identifying, localizing and quantifying the incidence and extent of coronary artery disease is investigated. Specifically, the association between the incidence and extent of coronary artery disease and the alterations in the endocardial surface morphology is studied. The results of the proposed approach on 15 normal data sets, and 12 abnormal data sets exhibiting coronary artery disease with varying levels of severity are presented. Based on the characterization of the endocardial surface morphology using the Bag-of-Words features, a neural network-based classifier is implemented to test the effectiveness of the proposed morphological analysis approach. Experiments performed on a strict leave-one-out basis are shown to exhibit a distinct pattern in terms of classification accuracy within the cardiac segments where the incidence of coronary arterial stenosis is localized.
\end{abstract}

Keywords: Ventricular endocardial surface, cardiovascular CT, non-rigid shape analysis, Bag-of-Words.

\section{Introduction}

The clinically observed relationship between the incidence and severity of Coronary Artery Disease (CAD) and the structural alterations in the left ventricular endocardial surface has not yet been formally studied due to inherent limitations of conventional cardiovascular imaging technologies. Since CAD is a leading cause of morbidity and mortality worldwide, techniques that improve diagnostic and prognostic effectiveness 
have a potentially significant clinical impact. X-Ray Coronary Angiography (XRA) is an invasive technique that is a clinically accepted standard for assessment of vascular morphology and for quantifying the extent of vessel stenosis due to artherosclerotic plaque deposition. However, a comprehensive assessment of CAD necessitates a study of both, vascular morphology and cardiovascular function. Conventional myocardial functional assessment is based on a stress test that uses radionuclide or magnetic resonance (MR) perfusion imaging techniques. Since vascular morphology and cardiovascular function are imaged using separate modalities, the time and cost associated with a comprehensive assessment of CAD and the potential health risk to the patient associated with higher radiation doses are all significantly increased.

Computed Tomography Coronary Angiography (CTCA) is a non-invasive imaging technique that allows for robust quantification of vascular morphology and also has the potential for characterizing the atherosclerotic plaque composition [1]. When performed using a 320 Multirow-Detector Computed Tomography (MDCT) scanner, CTCA can yield images with an isotropic spatial resolution of $0.5 \mathrm{~mm}$ in a volumetric fashion. The resulting CTCA images, in addition to providing vascular morphology information, are capable of providing significant details about the endocardial surface structure, in particular, the structure of the trabeculae and papillary muscles.

We hypothesize that certain changes in the endocardial surface morphology bear a direct relationship to changes in cardiovascular function, i.e., the incidence and extent of stenosis in a specific coronary artery can be localized via analysis of morphological changes in the endocardial surface. The only known previous work along these lines is our previous work [11] which tackled this problem using two basic shape descriptors under the assumption that the endocardial surface could be treated as rigid, since all the images were taken at a relatively steady phase of the cardiac cycle. Although this work produced significant results, it had some inherent problems with regard to the selection of the shape descriptors. The two shape descriptors were proposed under the assumption of rigidity of the endocardial surface as observed in the MDCT images, i.e., the endocardial surface was assumed to be free of global deformation. Although the data was collected at $75 \%$ in the R-R cardiac cycle, i.e., at a relatively steady phase, the continuous motion of Left Ventricle (LV) demanded a more robust shape descriptor, i.e., one that is invariant to global deformation $[9,10]$. In recent years, descriptors that are invariant to isometric deformations of an underlying surface have been studied in the context of shape-based retrieval in image databases. However, the problem in directly using existing shape descriptors developed for content-based image retrieval is that, these shape descriptors are designed with the goal of differentiating between two distinct classes of objects, e.g., "humans" with different poses versus "dogs" with different poses. In contrast, our goal is to differentiate between pathologies within the same class of objects, e.g., classify a particular LV segment as "normal" or "diseased", using its surface morphological properties.

Interest point feature-based methods have been used extensively in various computer vision algorithms [13] owing to the success of the Scale Invariant Feature Transform (SIFT) features [12] and Speeded Up Robust Features (SURF) [15]. The most advantageous aspect of interest point feature-based approaches is their treatment 
of images as a collection of primitive elements, i.e., "visual words", and their natural progression to the use of well-developed methods from text search. The two prominent implementations of visual words as a backbone for shape description are Shape Google- a technique for shape-based search in large collections developed by Ovsjanikov et.al. [8] and, the work of Toldo et.al. [14]. By computing the frequency of the geometrical word occurrences in an image, a representation referred to as Bagof-Features (BOF) is constructed for non-rigid shape description. As an extension to the BOF approach, we propose a novel shape analysis-based approach, termed as Bag-of-Words (BOW), to quantify the relationship between the incidence, severity and localization of CAD and the structural alterations in the LV endocardial surface.

Various computer vision applications have shown that visually similar images tend to share similar BOF descriptors. This property is useful for detection and description of similar images in a large-scale image database. The shape analysis community, on the other hand, has taken a long time to adopt BOF- or BOW-based approaches due to the lack of efficient and robust feature descriptors similar to SIFT. Some of the important properties of SIFT features include their inherent discriminative power combined with robustness to various image transformations. While several works in the research literature have proposed feature-based techniques for characterization of rigid shapes, very few are capable of dealing with non-rigid shape deformations [16]. To the best of our knowledge, this paper represents one of the first attempts, within the cardiovascular imaging community, to employ a BOW feature-based approach to compare non-rigid deformable shapes.

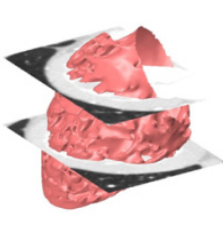

(a)

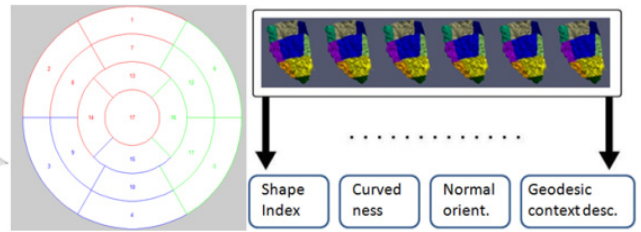

(b)

(c)

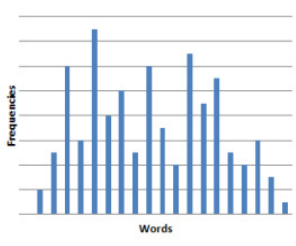

(d)

Fig. 1. Illustration of the sequence of steps in the morphological analysis of the LV endocardial surface: (a) accurate mesh segmentation, (b) generation of a 17-segment LV surface model with demarcation of coronary arterial territories (red: $L A D$, green: $L C X$, blue: $R C A$ ), (c) feature vector generation and $(\mathrm{d})$ generation of the BOW histogram via vector quantization $(K$-means clustering).

We have proposed and implemented a BOW feature-based approach to encapsulate the local and global geometry as well as the local orientation information of the LV endocardial surface within a robust feature vector for the purpose of morphological analysis. The experimental results show successful localization of coronary arterial stenosis and thereby serve to strengthen the clinically observed relationship between the incidence and severity of CAD and alterations in the LV endocardial surface. The sequence of steps in the proposed approach for morphological analysis of the LV endocardial surface is depicted in Figure 1.

The remainder of the paper is organized as follows. In Section 2, the proposed LV surface segmentation and LV shape analysis procedures are detailed; in Section 3, 
experimental results on the MDCT data sets are presented; in Section 4, the paper is concluded with a brief discussion of the proposed approach and an outline of directions for future work.

\section{MDCT Image Segmentation and LV Shape Analysis}

\subsection{Left Ventricle Segmentation and Meshing}

Three types of trabeculae morphologies are observed along the LV endocardial surface: (a) those that lie along the entire length of the ventricular wall forming prominent ridges; (b) those that are fixed at their extremities but free in the middle; and (c) those that connect the root of the papillary muscles and the ventricular wall. These trabeculae morphologies result in a complex endocardial surface topology. In order to adapt to the topological changes caused by the complex trabeculation structure, a 3D level set approach is employed to segment the LV endocardial surface. In order to suppress noise while still retaining the edges in the MDCT images, a median filter-based denoising procedure is employed on the 3D MDCT data prior to segmentation. The size of the median filter is empirically set to $7 \times 7$ based on the MDCT data set. A level set-based segmentation procedure without reinitialization, as proposed by Li et al. [5], is applied to the median-filtered 3D image data set followed by the marching cubes procedure [17] to generate the surface meshes. The surface meshes are subsequently denoised via a mean face normal filtering procedure proposed by Zhang and Hamza [7] to obtain the smooth shape of the myocardial surface of the left ventricle.

\subsection{Data Preparation}

In order to facilitate better understanding and localization of cardiac anatomy and pathology, the American Heart Association (AHA) has published recommendations for standardized myocardial segmentation [6]. We have adapted the AHA-approved 17-segment cardiac model [2] to divide the LV into 17 segments for more accurately localized shape analysis. The long axis of the LV is first computed, followed by division of the LV into 4 main parts, i.e., apex, apical, mid-cavity and basal along the longitudinal orientation. Division of the endocardial surface in the short axis view is tackled by exploiting knowledge of cardiac anatomy. Three landmark points are considered across the septum based on which the apical is divided into four parts and the mid-cavity and basal into six parts. Finally, the LV endocardial surface is divided into 17 segments.

\subsection{Feature Description}

500 surface points are randomly sampled from each of the $17 \mathrm{LV}$ endocardial surface segments. Four types of surface descriptors are considered, inspired by Toldo et.al. 
[14], to represent each sampled point. The first three descriptors are local whereas the fourth is a contextual descriptor. The descriptors are:

Shape Index $\left(I_{p}\right)$ : The shape index $I_{p}$ of a surface point $p$, as originally proposed by Koenderink [3] and subsequently modified by Zaharia and Preteux [4], is defined as a function of the two local principal surface curvatures $k_{p}^{1}$ and $k_{p}^{2}$ associated with surface point $p$ and formulated as follows:

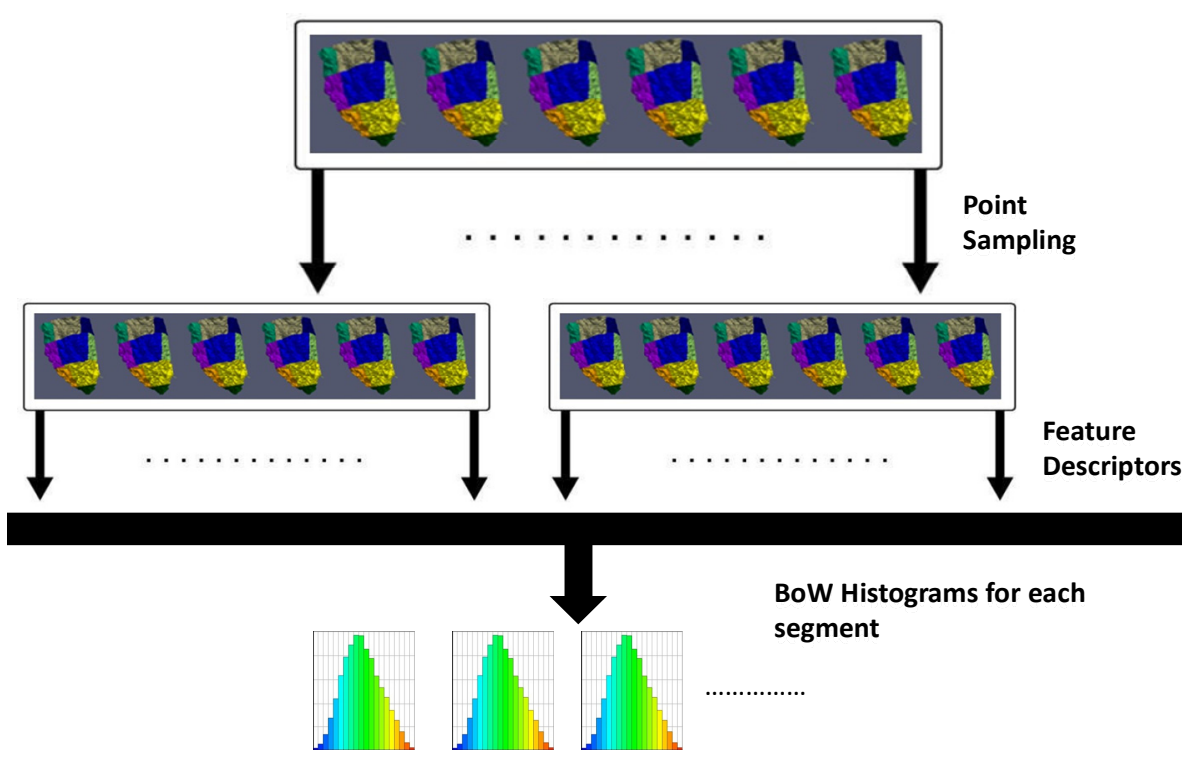

Fig. 2. Illustration of Bag-of-Words shape description and vector quantization

$$
I_{p}=\frac{1}{2}-\frac{1}{\Pi} \arctan \left(\frac{k_{p}^{1}+k_{p}^{2}}{k_{p}^{1}-k_{p}^{2}}\right)
$$

Where, $k_{p}^{1}>k_{p}^{2}$.

The range of the $I_{p}$ values is $[0,1]$. The value of $I_{p}$ is not defined for planar surfaces. The shape index $I_{p}$ provides a scale for representing basic elementary surface shapes such as convex, concave, rut, ridge and saddle [4]. The $I_{p}$ value is invariant to scale and $3 \mathrm{D}$ rigid-body transformations (i.e., translation and rotation) in Euclidean space. 
Curvedness $\left(C_{p}\right)$ : The curvedness $C_{p}$ of a surface point $p$, as proposed by Koenderink [3], is defined as a function of the two local principal surface curvatures $k_{p}^{1}$ and $k_{p}^{2}$ associated with surface point $\mathrm{p}$ and formulated as follows:

$$
C_{p}=\sqrt{\left(k_{p}^{1}+k_{p}^{2}\right) / 2}
$$

where, $k_{p}^{1}>k_{p}^{2}$.

Normal Orientation $\left(\theta_{p}\right)$ : The normal orientation $\theta_{p}$ at surface point $p$ is defined as the angle between the unit normal vector at surface point $p$ and the $X Z$-plane.

Geodesic Contextual Descriptor $\left(G C D_{p}^{20}\right)$ : The geodesic contextual descriptor, denoted by $G C D_{p}^{20}$, is a contextual descriptor that depends on the relative position of the surface point $p$ vis-à-vis the other points on the surface segment. It is characterized by a 20 -bin histogram which is generated by computing the normalized geodesic distance between the surface point $p$ and 499 other sampled points on the surface segment. The geodesic contextual descriptor $G C D_{p}^{20}$ is scale-invariant.

The feature extraction procedure described above results in a 23 -tuple feature vector for each surface point $p$ denoted by $F_{p}=\left(I_{p}, C_{p}, \theta_{p}, G C D_{p}^{20}\right)$ as depicted in Figure 2.

\subsection{Construction of Visual Vocabularies}

The feature vectors $F_{p}$ at each surface point are clustered in order to obtain the visual words. Assuming that the local descriptors are computed for a set of stable surface points, we quantize the feature vector space to obtain a compact representation for the vocabulary of visual words, in a manner similar to the Shape-Google approach [8]. A vocabulary is defined as a set of representative vectors in the descriptor/feature space, obtained by means of unsupervised learning, i.e., vector quantization via $k$-means clustering. More formally, a vocabulary is defined as a collection $V=\left\{v_{1}, \ldots, v_{k}\right\}$ where $v_{i}$ is the centroid of the $i^{\text {th }}$ cluster and the clusters represent the visual words. Here, we have chosen $k=20$ in the $k$-means clustering algorithm for generating the final histogram.

\section{Experimental Results}

We employed the proposed methods for segmentation, meshing and endocardial surface shape description on 27 MDCT data sets consisting of 12 data sets from cardiac patients and 15 data sets from normal subjects. Incidence of single- or multivessel obstructive CAD was found in the three major coronary arteries using XRA, which was further confirmed by myocardial perfusion and fractional flow reserve tests performed on the patients. 
The cardiac patients and normal subjects were subject to a contrast-enhanced CTCA scan on a 320-MDCT scanner using a standard CT angiography protocol with ECG gating. The resulting images were reconstructed at $75 \%$ in the R-R cardiac cycle to ensure minimal ventricular motion, so that the subsequent shape analysis is minimally affected by cardiac motion. The segmentation method described in Section 2.1 was used to generate topologically correct and geometrically accurate data.

\subsection{Segmentation Results}

The results of the LV segmentation have already been proven to be reasonably accurate [11]. Additionally, the spatial distribution of the trabeculation was observed to vary with the location within the LV; thus providing the rationale for using the 17segment AHA model to perform localized shape analysis. Furthermore, the proposed segmentation approach has already shown a visually observable distinction in trabeculation between normal and diseased hearts, yielding classification accuracy greater than $80 \%$ with simple rigid shape descriptors and a nearest-neighbor classifier [11]. Our previous work has demonstrated the accuracy of the proposed segmentation method as well as its applicability for subsequent quantitative shape analysis.

\subsection{Localization Results}

A coronary artery is considered as diseased or stenotic if the extent of stenosis is $50 \%$ or greater. The LV myocardial segments are labeled as diseased by a cardiologist if they are supplied by stenotic arteries. An artificial neural network (ANN), employing a multilayer perceptron (MLP) architecture with a single hidden layer and a learning rate of 0.3 , is used for the purpose of classification of the LV segments. The 20-bin histograms, generated via the vector quantization procedure (Section 2.3), for a particular LV segment from all the LV datasets are used as the inputs to the ANN. The classification procedure is carried out within a strictly leave-one-out setting. The output of the ANN is whether a particular LV segment can be classified as "normal" or "diseased".

The success rate for detection of stenosis in a specific coronary artery is shown in Figure 3. The classification results depict a clinically observed and intriguing relationship between the coronary arterial stenosis and the affected segment in the 17segment AHA model. The lower classification accuracy in the basal area (segments 16) may be explained by the clinical observation that many of the coronary arterial stenoses in this study are located in the mid to distal portion of the coronary arteries that only effect the mid-cavity (segments 7-12) and apical portions (segments 13-16) of the LV endocardial surface. Furthermore, another probable reason for the lower classification rate in the basal area is that the apical and mid-cavity segments exhibit greater endocardial trabeculation structure than the basal segments, which translates to more reliable endocardial surface morphology information that can be used for the purpose of classification. 

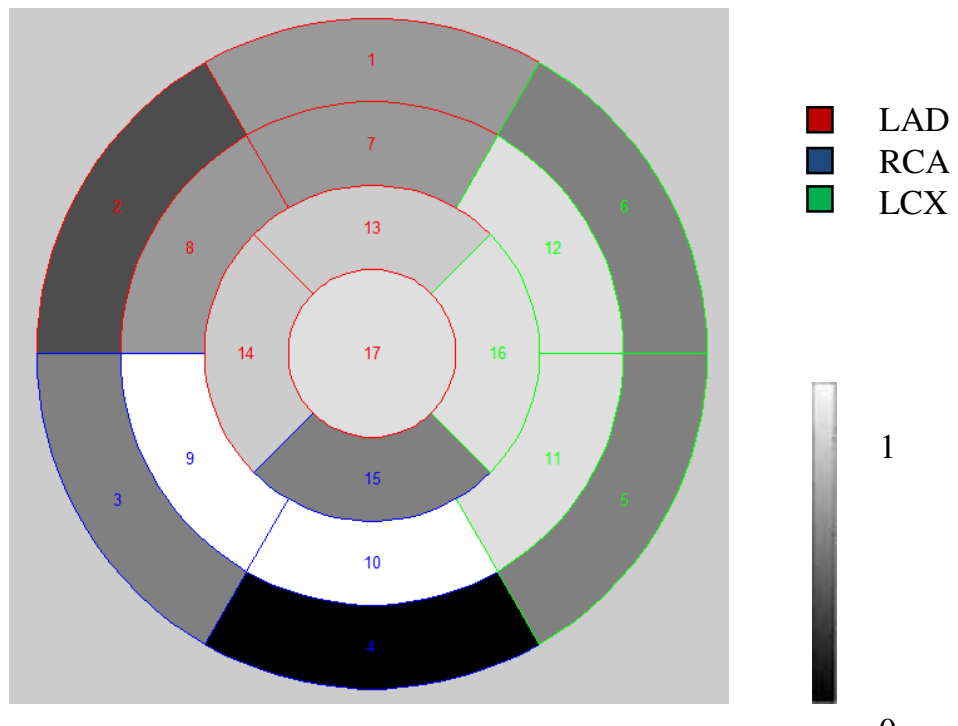

0

Fig. 3. Illustration of the classification accuracy for detection of coronary artery stenosis based on the change of surface morphology in the 17 LV segments in the AHA model. Higher gray values denotes higher classification accuracy and vice versa.

\section{Discussion and Conclusions}

To the best of our knowledge, this paper is one of the earliest works that studies the relationship between coronary artery stenosis and the morphological alterations in the LV endocardial surface using high-resolution MDCT data, and demonstrates its potential predictive value for the incidence and severity of CAD. This investigation also sheds new light on the localization of LV regions that are the most affected by coronary artery stenosis, a phenomenon which is yet to be fully explained. This association between the morphological features of the endocardial surface and cardiac functionality will be further explored in our future work. In particular, we aim to investigate the correlation between the endocardial surface morphology and the results of myocardial perfusion and fractional flow reserve tests in addition to the coronary arterial stenosis results obtained via XRA.

\section{References}

[1] Goo, S., Joshi, P., Sand, G., Gerneke, D., Taberner, A., Dollie, Q., LeGrice, I., Loiselle, D.: Trabeculae carneae as models of the ventricular walls: implications for the delivery of oxygen. Jour. Gen. Physiology 134(4), 339-350 (2009)

[2] Cerqueira, M.D., Weissman, N.J., Dilsizian, V., Jacobs, A.K., Kaul, S., Laskey, W.K., et al.: Standardized myocardial segmentation and nomenclature for tomographic imaging of the heart. Circulation 105, 539-542 (2002) 
[3] Koenderink, J.: Solid Shape. The MIT Press, Cambridge (1990)

[4] Zaharia, T., Preteux, F.: 3D Shape-based retrieval within the MPEG-7 framework. In: Proc. SPIE Conf. Nonlinear Image Proc. Pattern Anal. XII, vol. 4304, pp. 133-145 (2001)

[5] Li, C., Xu, C., Gui, C., Fox, M.D.: Level set evolution without re-initialization: a new variational formulation. In: Proc. IEEE Conf. CVPR, vol. 1, pp. 430-436 (2005)

[6] Medtronic Inc, The Visible Heart webpage, http: / /www.visibleheart.com/index.shtml

[7] Zhang, Y., Hamza, A.B.: Vertex-based diffusion for 3-D mesh denoising. IEEE Trans. Image Processing 16(4), 1036-1045 (2007)

[8] Ovsjanikov, M., Bronstein, A.M., Bronstein, M.M., Guibas, L.J.: Shape Google: a computer vision approach to invariant shape retrieval. In: Proc. NORDIA Workshop (2009)

[9] Sun, J., Ovsjanikov, M., Guibas, L.J.: A concise and provably informative multi-scale signature based on heat diffusion. In: Proc. Symposium on Geometry Processing (2009)

[10] Mémoli, F., Sapiro, G.: A theoretical and computational framework for isometry invariant recognition of point cloud data. Found. Comput. Math. 5(3), 313-347 (2005)

[11] Mukhopadhyay, A., Qian, Z., Bhandarkar, S., Liu, T., Voros, S.: Shape Analysis of the Left Ventricular Endocardial Surface and Its Application in Detecting Coronary Artery Disease. In: Metaxas, D.N., Axel, L. (eds.) FIMH 2011. LNCS, vol. 6666, pp. 275-283. Springer, Heidelberg (2011)

[12] Lowe, D.: Distinctive image features from scale-invariant keypoints. Intl. Jour. Computer Vision 60(2), 91-110 (2004)

[13] Matas, J., Chum, O., Urban, M., Pajdla, T.: Robust wide-baseline stereo from maximally stable extremal regions. Image and Vision Computing 22(10), 761-767 (2004)

[14] Toldo, R., Castelllani, U., Fusiello, A.: The bag of words approach for retrieval and categorization of 3D objects. The Visual Computer 26(10), 1257-1268 (2010)

[15] Bay, H., Ess, A., Tuytelaars, T., Van Gool, L.: SURF: speeded up robust features. Computer Vision and Image Understanding (CVIU) 110(3), 346-359 (2008)

[16] Mitra, N.J., Guibas, L.J., Giesen, J., Pauly, M.: Probabilistic fingerprints for shapes. In: Proc. Symp. Geometry Processing, Vienna, Austria (2005)

[17] Lorensen, W.E., Cline, H.E.: Marching cubes: A high resolution 3D surface construction algorithm. Computer Graphics 21(4), 163-169 (1987) 